\title{
DIE IDEOLOGISCHE ORIENTIERUNG DER NEUEN STAATEN IN ÜBERSEE
}

\author{
Von Herbert KRÜGer*
}

In den neun Jahren ihrer Wirksamkeit hat sich diese Zeitschrift bemüht, im einzelnen und vergleichend $\mathrm{zu}$ erkennen, in welche Verfassungen, von der politischen Konstituierung angefangen, sich die Neuen Staaten in Übersee zu bringen suchen, um ihren Entschluß zur Modernisierung so erfolgreich wie möglich verwirklichen zu können. Mit diesem Angang ist vorausgesetzt, daß es diesen Staaten nicht um eine abstrakt ideale oder gar utopische Verfassung geht, daß sie vielmehr als Verfassunggeber aus einer selbstgeschaffenen Lage, eben dem Entschluß zur Modernisierung, und aus den Umständen, unter denen er durchgesetzt werden muß, also kurz gesagt „realpolitisch“ argumentieren. So wichtig diese Einsicht ist und so sehr sie um der Vermeidung von "Idealpolitik“ willen festgehalten werden muß, so erschöpft sie doch den Vorgang des Sich-in-Verfassung-Bringens mit dem Ziele der Modernisierung nicht. Auch hier wiederum ist vielmehr $\mathrm{zu}$ berücksichtigen, daß Verfassungen zwar in Auseinandersetzung mit den konkreten Situationen (zu denen auch die nationalen Ziele gehören) geschaffen werden, daß diese Auseinandersetzung aber nicht bloße Reaktion, sondern Selbständigkeit und Eigenart als wesentliche, wenn nicht sogar entscheidende Elemente in den Prozeß der Konstituierung der Nation und ihrer Verfassung einführt. So sehr also Menschen und Völker von den Notwendigkeiten der Sache und von den „Forderungen des Tages“ bestimmt sein mögen - ihre Antwort auf alle solche Herausforderungen bleibt doch immer eine autonome und muß eine solche bleiben, wenn man den Menschen und Völkern Freiheit und Würde zuerkennt. Damit wird die Geistesverfassung oder wie man immer diese Kraft nennen mag (Genius der Nation, Volksgeist, political culture u. a. m.) als diejenige Kraft erkannt und gewürdigt, die letzen Endes alle Verfassunggebungen bestimmt.

Der Beginn des zehnten Jahrganges dieser Zeitschrift verpflichtet dazu, diesem innersten Gegenstand ihrer gesamten Thematik wenigstens einen Versuch zu widmen. Wir greifen aus diesem Komplex dasjenige Element heraus, das verhältnismäßig noch am ehesten ans Tageslicht zu fördern und in den Griff $z u$ bekommen ist. Wenn wir nämlich bei der Geistesverfassung der Neuen Nationen ansetzen, so wird hiermit nicht etwa eine vorgegebene, statische, lediglich bestimmende Größe angesprochen. Die Geistesverfassung ist vielmehr selbst wieder eine immer wieder zu bewirkende Hervorbringung der Gruppe. Zwar ist die Fruchtbarkeit des Geistes das Geschenk eines Unbekannten - das große Rätsel, warum es schöpferische und sterile Perioden im Leben der Völker gibt. Aber die Völker können solche Sternstunden auch verkennen und ungenutzt lassen. Wenn also so viel und so oft beschworene Eigenschaften wie Offenheit und Wachsamkeit irgendwo einen Sinn haben, dann liegt er an dieser Stelle. Daß die Erlangung der Selbständigkeit ein geschichtlicher Höhepunkt ist, der fruchtbar gemacht werden kann, unterliegt keinem Zweifel: Der Übergang von kolonialer Unterworfen-

\footnotetext{
* Seinem lieben Freunde Hans Möller zum siebzigsten Geburtstag am 3. März 1977 gewidmet
} 
heit $\mathrm{zu}$ verantwortungsvollster Selbständigkeit und deren heroische Erkämpfung bilden Ereignisse von einer Geschichtsmächtigkeit, die an sich imstande sind, schöpferische Kräfte zu entbinden und den Neuen Staaten durch das Ergreifen einer solchen Stunde zu einer eigenartigen Identität zu verhelfen.

Nach mancherlei Jahrzehnten auch der geistigen Fremdherrschaft scheint es nahezuliegen, dieses Selbst durch Rückzug auf sich selbst zu gewinnen - womöglich durch Rückkehr zu vorkolonialen Mentalitäten. Es wird gezeigt werden, daß von unbedeutenden Ausnahmen abgesehen - ein solcher Entschluß in keinem der Neuen Staaten gefaßt worden ist. Es wäre dennoch denkbar, daß man die neue, moderne Orientierung ausschließlich aus der eigenen moralischen und intellektuellen Substanz zu gewinnen suchte - allenfalls im Austausch miteinander (sc. der dekolonisierten Nationen), aber nicht in Auseinandersetzung mit "Ost" oder „West". Eine solche Beschränkung zwar nicht auf die eigene Vergangenheit, wohl aber auf die nationale Eigenart scheint sich nicht zuletzt deswegen zu empfehlen, weil die Auseinandersetzung mit oder gar die Übernahme von westlichen oder östlichen Ideen als Stellungnahme für einen der großen weltpolitischen Blöcke mißdeutet werden könnte, obwohl sich die Neuen Staaten aus deren Konfrontation gerade heraushalten wollen. Dieses Risiko muß dennoch gewagt werden, und zwar aus zwei Überlegungen. Es mag einmal die geistige Fruchtbarkeit der Neuen Staaten so groß sein, wie nur denkbar - es ist erst die Erprobung am Maßstab anderer Ideen, die überzeugend die Richtigkeit des eigenen Denkens dartut. Und zweitens: Wenn die eigene Fruchtbarkeit nicht so unerhört ist, wie sie sein könnte - ganz abgesehen davon, daß niemand mehr irgendwo ab ovo geistig zu beginnen vermag -, dann bedarf es zum mindesten für eine Übergangszeit der Übernahme fremden Geistesgutes. Da es aber außerhalb der Dritten Welt nennenswerte geistige Bestände außer Ost und West nicht gibt, kann die Auseinandersetzung mit ihnen nicht umgangen werden, es sei denn, die Dritte Welt wolle sich von vornherein mit geistigem Provinzialismus begnügen.

Es scheint, als wenn die Neuen Staaten sich jedenfalls zur Zeit in dem zweiten Falle befänden. Zwar hat man sich zur Modernität entschlossen, aber für Neophyten ist es schwierig, sich in der Modernität zurechtzufinden. Was hat man zu tun oder auch zu lassen, um äußerlich und innerlich modern zu werden: Soll man sich für den Primat der Industrialisierung entscheiden oder vor allem erst die Ertragskraft der Landwirtschaft steigern? Was Albert O. Hirschmann ${ }^{1}$ vor vielen Jahren beobachtet hat, dürfte heute noch gelten: Es fehlt hier noch an der Fähigkeit, die richtigen "developments decisions“ zu treffen, an dieser Stelle ist daher vor allem Orientierung vonnöten. Unter dem Blickwinkel der Entwicklungshilfe ergibt sich nichts anderes. An ihr hat man wohl mit Recht kritisiert das Übergehen der „political gaps“2. Damit ist angesprochen die Notwendigkeit, daß Modernisierung feste und leistungsfähige Institutionen voraussetzt. Gerade hieran aber fehlt es nach einer immer wieder bekundeten Auffassung in den Entwicklungsländern; insbesondere: Was nützt alle Planung, wenn es nicht gelingt, die Durchsetzung eines Planes, und zwar nachhaltig zu sichern? Es folgt hieraus, daß ebensowenig wie eine Nation ihre Geistesverfassung in esoterischer Meditation zu

\footnotetext{
1 The Strategy of Economic Development (Yale UP 1958, siebzehnter Druck 1975), S. 25 f.

Zur Frage der Notwendigkeit einer Ideologie für die neuen Staaten vgl. Alexander B. Woodside

2 Vgl. Samuel P. Huntington, Political Order in Changing Societies (Yale UP 1968), S. 5
} 
gewinnen vermag, die Entwicklungshilfe sich auf finanzielle oder technische Unterstützung beschränken darf: Selbstverständlich soll sie nicht inzidenter Ideologien imputieren. Aber sie sollte Ideen, Modelle, Gesichtspunkte u. a. m. mehr in sich einbeziehen, damit jedenfalls Gegenpositionen geboten werden, die den Neuen $\mathrm{Na}$ tionen dazu verhelfen, in Auseinandersetzung mit ihnen, die durchaus auch mit einer Verwerfung ausgehen mag, zu sich selbst zu finden. Es handelt sich also m. W. darum, den Neuen Staaten möglichst viele und verschiedene Topoi vorzustellen. Es ist dies allerdings ein modus procedendi, der den Neuen Staaten fremd erscheinen mag ${ }^{3}$. Es müßte daher dieser Modus des Geistig-zu-sich-selbst-Findens erst rezipiert werden, um sie in die Lage zu versetzen, in solcher Diskussion ihre Eigentümlichkeit $\mathrm{zu}$ erarbeiten und $\mathrm{zu}$ sich selbst $\mathrm{zu}$ finden - als die Voraussetzung aller Voraussetzungen überhaupt.

Mit der Entscheidung dafür, eine ideologische Identität durch Auseinandersetzung zu gewinnen, hat man sich somit zugleich für Rezeption entschieden, gleichgültig, ob man im Endergebnis fremde Ideen übernimmt oder verwirft. An sich müßte daher einer Behandlung des Themas „ideologische Orientierung“ eine Theorie der Rezeption vorausgeschickt werden. Dies ist schon deswegen nicht möglich, weil es eine voll ausgebildete Erkenntnis dieses Phänomens nicht gibt, diesem Mangel aber nicht inzidenter abgeholfen werden kann ${ }^{4}$. Es muß hier genügen, wenn zweierlei aus diesem Komplex herausgehoben und klargestellt wird.

Als erstes muß das zu übernehmende Geistesgut übernehmbar sein. Damit ist einmal gesagt, daß es sich muß von dem Boden lösen lassen, aus dem es erwachsen ist, und zum zweiten muß es sich in eine ganz andere Umwelt einwurzeln lassen. Denkt man hierbei an Verfassungen, so sind offenbar flexible Konstitutionen zur Übertragung geeigneter als rigide - nebenbei vielleicht nicht der letzte Gesichtspunkt, der für eine Flexibilität der Verfassung spricht. Was die Einwurzelung in einer neuen Umwelt angeht, so bedeutet sie in jedem Falle Umwandlung im Sinne dieser Umwelt: Das römische Recht ist in Deutschland nicht als klassisches, sondern im "usus modernus" zur Anwendung gelangt, und als im 19. Jahrhundert die Historische Rechtsschule die Rückkehr zum Klassischen Römischen Recht durchsetzte, bereitete sie damit der praktischen Anwendbarkeit dieses Rechtes ein Ende.

Unter diesem Blickwinkel lassen sich Zweifel an der Rezipierbarkeit der östlichen und der westlichen Ideologie in die Dritte Welt nicht unterdrücken. Der Marxismus-Leninismus ist eine geschlossene, endgültige, jederzeit und überall gleich verbindliche'Heilslehre, die Variationen gemäß konkreten Umständen nicht gestattet. Bezeichnend hierfür ist der Streit darüber, ob ein „Nationaler Weg“ zum Sozialismus zulässig sei: Wenn dergleichen auch immer wieder von Jugoslawien und den westlichen kommunistischen Parteien gefordert wird, so kann doch kaum ein Zweifel daran bestehen, daß die Ideologie einen solchen „Polyzentrismus“5 nicht einschließt, wie insbesondere die letzte Konferenz dieser Parteien gezeigt hat, indem sie sich zu dem „Proletarischen Internationalismus“ bekannte". Die

\footnotetext{
3 Wie insbesondere ihre Verständnislosigkeit für jede Art von „Opposition“ zeigt; dazu unten.

4 Einen bedeutenden Fall von Rezeption, die des Code Čivil in Westdeutschland, behandelt Elisabeth Fehrenbach, Traditionelle Gesellschaft und revolutionäres Recht (Göttingen 1974)

Wichtige Elemente zu einer Theorie der Rezeption enthält die Schrift Brun-Otto Bryde's, The Politics and Sociology of African Development (Frankfurt am Main 1976).

5 Dieser Terminus ist von dem Führer der. KPI, Togliatti - geprägt worden; vgl. W. Laqeur and

L. Labedz, Polycentrism - The New Factor in International Communism - (New York 1962).

6 Sie hat in der Mitte des Jahres 1976 in Berlin (Ost) stattgefunden.
} 
östliche Literatur, die sich mit den Neuen Staaten beschäftigt, stellt sie folgerichtig vor die Alternative, sich auf marxistisch-leninistische Art und Weise zu modernisieren oder überhaupt nicht $\mathrm{zu}$ modernisieren, da es einen dritten Weg in die Zukunft nicht gebe ${ }^{7}$.

Man mag es als einen Fall von „Konvergenz" deuten, daß Verhärtung zwar nicht eine angeborene, wohl aber eine erworbene Eigenschaft der westlichen Ideologie ist. Was im Osten von Anfang an der Fall gewesen ist, ist im Westen eine Alterserscheinung: Die Erhebung, oder richtiger Pervertierung, einer Weltanschauung zu einer säkularen Religion. Es haben dies vor allem die beiden Weltkriege gezeigt, insofern sie im Namen religiöser und sittlicher Ideale, die wie z. B. „Demokratie" zugleich oder im Grunde politische sind, geführt worden sind. In Gestalt des Neoliberalismus hat sogar so etwas Profanes wie die Wirtschaftsverfassung sakrale Züge angenommen: Es zeigt sich dies vor allem darin, daß er dem Westen sonst so wesentliche "Geist der Prüfung " 8 von dieser Materie aus geschlossen ist, wie es für eine offenbarte Religion charakteristisch erscheint: Hier darf nicht geprüft oder gar kritisiert werden - hier sind allein Verkündung und Lobpreisung zulässig, wobei sich das paradoxe Bild religiöser Verkündung in den Handelsteilen liberaler Tageszeitungen ergibt.

$\mathrm{Da}$ es den Neuen Staaten nicht um die beste Religion, sondern um säkulare Modernisierung geht, erweisen sich alle Ideologien als unübertragbar und unübernehmbar, die zu Religionen denaturiert sind. Hiernach ist für uns eine zweite Überlegung aus dem großen Komplex „Rezeption“ wichtig. Gerade wenn es wie hier um Modernisierung geht, sind ausgeschlossen von der Rezeption Ideologien, deren Modernität nicht über jeden Zweifel erhaben ist. Etwas Derartiges steht gerade auch für die östliche Ideologie zu befürchten, obwohl sie sich selbst als die im Vergleich zum Westen fortgeschrittenere betrachtet. Diese Zweifel gelten als erstes dem "Glück“, das der Osten im staats- und herrschaftslosen, „paradiesischen“ Zustand verheißt ${ }^{9}$ : Sosehr sich gerade der Osten über alles „Kleinbürgerliche“ erhebt, so stellt sich dieses Glück als das Kleinbürgertum in Reinkultur dar, womit zugleich jeder geistigen Fruchtbarkeit das Urteil gesprochen ist. Auch und gerade an dieser Stelle wieder besteht für den Westen keine Veranlassung zu Selbsterhebung: Es genügt ein Blick auf das "Glück“, auf das die Wohlstands- und Genußgesellschaft hinauswill: Es ist für jedermann dasselbe und daher entsprechend konfektioniert. Zwar wollen und sollen auch die Neuen Staaten die Armut überwinden und jedermann eine ausreichende Lebensgrundlage verschaffen. $\mathrm{Ob}$ dies aber das letzte und endgültige Wort meinen kann, muß als unsicher bezeichnet werden: Wenn insbesondere "Humanismus" gehuldigt wird, dann kann er nicht das standardisierte kleinbürgerliche Glück der großen Zahl im Sinne haben. Läßt man die Vorstellung von "Glück“ auf sich beruhen, dann stellt sich die Frage nach dem Weg, der zu dem Ziele der Modernität führen soll. Der Osten hält den Klassenkampf für die einzige Möglichkeit, die es in dieser Hinsicht gibt. Ihm stimmen diejenigen, allerdings nicht allzu zahlreichen Autoren zu, die Existenz

\footnotetext{
7 Vgl. z. B. Günter Barthel, Industrialization in the Arab Countries of the Middle East (Berlin 1972).

$8 \mathrm{Vgl}$. hierzu das Bundesverfassungsgericht der BRD in ständiger Rechtsprechung: Freie Meinungsäußerung und geistige Auseinandersetzung sind für die freiheitliche Demokratie schlechthin konstituierend; zuletzt etwa die Entscheidung vom 28. April 1974, in: BVerfGE 42.139.

9 Vgl. J. M. Gillisoon, The Soviet Image of Utopia (The John Hopkins U.P. 1975).

A. J. Gregor, The Fascist Persuasion in Radical Politics (Princeton U.P. 1974).
} 
von Klassen in den neuen Staaten vermissen und auf ihr Hervortreten drängen ${ }^{10}$. Gleichgültig ob Klassenkampf, Diktatur des Proletariats usw. im 19. und noch im 20. Jahrhundert unumgänglich gewesen sind oder nicht: Selbst wenn man die Ideale des Marxismus-Leninismus bejaht, zwingt nichts dazu, für den Weg, der zu ihnen führt, nichts aus der Geschichte zu lernen und sie weniger aufwendig und gewaltsam anzustreben. Für Marxismus-Leninismus sind Klassenstruktur und die Modalität des Kampfes ein vorübergehender, zu überwindender Zustand, und wenn im Osten schon seit langem vom „Staat des ganzen Volkes“ die Rede ist, dann deutet dies darauf hin, daß Klassenkampf nur noch in den auswärtigen Verhältnissen stattfindet. In dieser Sicht muß diejenige Strategie die beste sein, die dem Mangel an Klassen nicht etwa durch Bildung von solchen abhilft, um dem Gesetz zu genügen, sondern die im Gegenteil deren Bildung verhindert und von vornherein von einer klassenlosen Gesellschaft ausgeht ${ }^{11}$. Anzeichen für eine solche Strategie deuten sich darin an, daß man vielfach der Bildung einer neuen Elite entgegentritt, die sich an die Stelle der Kolonialherren setzt und die Herrschaft ebenso wie diese in ihrem Sinne ausübt ${ }^{12}$.

Aber auch die Wege, die nach Ansicht des Westens allein zur Modernität führen, sind für die Neuen Staaten nicht ohne weiteres annehmbar oder gangbar. Das gilt als erstes für die Auffassung, daß nur die Freiheit des Individuums sowohl das Glück des einzelnen wie vor allem auch der Gesamtheit zu bewirken vermag. Es trifft dies aber auch zu für die Überzeugung, daß es allein der Wettbewerb zwischen solchen Individuen ist, der die Freiheit zu solchen Erfolgen steuert. In Asien und in Afrika wird nach wie vor hochgehalten das Ideal, daß der Mensch sich nicht als Individuum, sondern als Mitglied einer Gemeinschaft erfährt und erfüllt. Das gilt insbesondere auch für die Agrarverfassung. Und was den Wettbewerb als Demiurgen angeht, so muß man sich fragen, ob er an der entscheidenden Stelle, nämlich in der Wirtschaft, noch als diejenige "Wunderwaffe" wirkt, als die man ihn heute noch betrachtet ${ }^{13}$, und ob er daher diejenigen Nationen, die dieser Denkfigur unbefangen gegenüberstehen, so sehr überzeugt, daß sie nicht umhin könnten, ihn schleunigst und rein zu übernehmen.

Insgesamt gilt: Wer sich geistig erneuern will, wird Hilfe hierzu nicht gerade dort suchen, wo man selber erneuerungsbedürftig erscheint. Zweifel daran, ob Rezeption in diesem Sinne fruchtbar ist oder nicht, werden sich vor allem dann einstellen, wenn die zur Rezeption gestellten geistigen Bestände von ihren Trägern selbst nicht mehr vorbehaltlos und unbedingt bejaht werden. Derartiges trifft zwar nicht für den Osten zu, dies aber lediglich deswegen, weil dort Zweifel an der Ideologie nicht geduldet werden. Es gehört zu den Vorzügen des Westens, daß dort Zweifel nicht unterdrückt, sondern gehegt werden. Beschränken wir uns hiernach auf den Westen, so sind es zwei Symptome, die eine Rezipierbarkeit seiner Ideologie durch

10 Vgl. P.-E. Gonidec, Les Systèmes Politiques Africaines II (Paris 1974), S. $204 \mathrm{ff}$.

Robert Martin, Personal Freedom and the Law Tanzania (Nairobi Oxford U.P. 1974).

Issa G. Shivji, Class Struggle in Tansania (London 1976), Besprechung in International Affairs 1976, S. 664

11 Gegen die Bildung von Klassen Leopold Senghor nach I. L. Markovitz, Senghor and the Politics of Negritude (London o. J.), S. 149: „Functionally, the doctrine of community attempts to prevent the development of class consciousness, of the transformation of a , class in itself ' to a ,class for itself'“; development of class consciousness, of the transformation of a , class in itself' to a , class for itself'“;
hierzu ferner a. a. O. 124: In Senegal gibt es keine Klassen; 139: Auch im vorkolonialen Senegal hat es Klassen nicht gegeben; 145: "African society is not simply a classless society; more positive, it is a

12 Vommunity society".

13 Allerdings ist es heute weithin der Staat, der ihn durch Verbote von wettbewerbsbeschränkenden Abreden und durch Aufsicht über marktbeherrschende Unternehmen, insbesondere auch durch Untersagung von Fusionen, künstlich mit herrschaftlichen Mitteln am Leben zu halten sucht. 
die Dritte Welt unter diesem besonderen Gesichtspunkt als zweifelhaft erscheinen lassen.

Es handelt sich einmal um die These, daß der Westen an unfruchtbaren und unüberwindlichen Widersprüchen oder Konflikten leide, an denen er auf die Dauer zugrunde gehen müsse. Zwar kann hiervon im Ernst nicht die Rede sein - man könnte eher beklagen, daß es ernsthafte Unterschiede und Gegensätze nicht mehr gibt und daß darunter die geistige Fruchtbarkeit des Westens kränkelt. Aber vielleicht liegt gerade hierin die Ursache dafür, daß man solche Elemente der Lebendigkeit konstruiert und durch Extremismus sterilisiert. Hier scheint ein Begriffsrealismus am Werke, der seine Prämissen aus der Luft greift und in allen nur denkbaren Kombinationen derselben in sich kreist: Man kann sich des Eindruckes eines "l'art pour l'art" nicht erwehren. Insbesondere wird auf das Beste, was der Westen zu bieten hat, nämlich ständig neue Erfassung der Wirklichkeit, Erneuerung ihres Bildes und entsprechende Berichtigung der Ideologie, verzichtet. Man begnügt sich mit dem Bild, das Marx entworfen hat, auf die Gefahr hin, daß es schon zu seiner Zeit nicht richtig gewesen ist. Es versteht sich von selbst, daß ein jenseits der Wirklichkeit geführtes Selbstgespräch von Intellektuellen für die neuen Staaten ohne jegliche Bedeutung ist und seine „Ergebnisse“ daher für eine Rezeption nicht in Frage kommen - ausgenommen vielleicht einige Studenten, die im Westen studiert haben und von dort nichts Besseres als solchen Begriffsrealismus mit nach Hause gebracht haben.

Es liegt auf der Hand, daß die neuen Staaten an der Übernahme einer Optik nicht interessiert sein können, die das menschliche Leben in Konflikte auflöst und sie daher dort substituiert, wo sie nicht angelegt sind. Handelt es sich für sie vor allem um Bildung einer Nation aus den verschiedensten und gegensätzlichsten Gruppen aller Art, dann ist für sie wichtiger eine Neutralisierung der Konflikte sowie die Pflege von Zusammenhang und Zusammenarbeit, zumal wenn sich vorgegebene Ansätze und Neigungen hierfür finden. Aber auch die Fruchtbarmachung solcher Konflikte durch Versetzung derselben in ein Verhältnis geordneten Wettbewerbes vermag wenig hilfreich zu erscheinen, zumal wenn der Westen selbst bisher nicht imstande gewesen ist, zu erklären, wodurch aus privaten Lastern öffentliche Tugenden hervorgehen müssen ${ }^{15}$.

$\mathrm{Zu}$ den Modalitäten, die die Rezeption einer Ideologie ausschließen oder jedenfalls erschweren, gehören auch deren Zweifel an ihr selbst. Gerade dies aber ist der Fall des Westens, insofern hier der Geist der Prüfung zu einer Raserei der Verneinung alteriert worden ist. Angesprochen ist hiermit eine Mode - anders kann man diese Strömung schwerlich charakterisieren —, die sich darin gefällt, die Legitimität alles Bestehenden in Zweifel zu ziehen. Es gibt so gut wie nichts, von dem nicht ein "Legitimitätsdefizit" oder gar „eine Legitimationskrise" behauptet würde ${ }^{\mathbf{1 4}}$. Solche Thesen laufen in jedem Falle darauf hinaus, daß alles — insbesondere auch die geistigen Bestände - , was besteht, wert ist, daß es zugrunde geht - also eine Existenz per nefas führt und der moralischen Sanierung durch eine neue Recht-

14 Vgl. statt vieler z. B. Rolf Ebbinghausen. Bürgerlicher Staat und politische Legitimation (Frankfurt am Main 1976). Peter Graf Kielmannsegg, Legitimationsprobleme politischer Systeme = Bericht über die Tagung der Deutschen Vereinigung für Politische Wissenschaft in Duisburg, Herbst 1975 (Opladen 1976). Für einen Autor ohne Tendenz vgl. Daniel Bell, The Cultural Contradictions of Capitalism (New York 1976), S. $180 \mathrm{ff}$.

15 Vgl. hierzu die Darstellung und Auseinandersetzung, die Fritz W. Scharpf, Politische Durchsetzbarkeit innerer Reformen (Göttingen 1974), S. $20 \mathrm{ff}$. gibt. Der unter dem Namen "Watergate“ bekannte große Skandal hat mit Grund in den Vereinigten Staaten das Vertrauen in die politischen Institutionen und Personen erschüttert, doch scheint man sich hier wieder gefangen zu haben. 
fertigung bedarf. Als Beispiel hierfür sei genannt das Wirtschaftsunternehmen: Das Eigentum ist angeblich nicht mehr imstande, es $\mathrm{zu}$ legitimieren - erst die paritätische Mitbestimmung wird diesen Mangel beheben (wie überall „Demokratisierung ") ${ }^{16}$.

Legitimation und Legitimität stellen zwar gerade auch für die Neuen Staaten wesentliche Probleme dar. Aber während man sich im Westen darin gefällt, sie zu zerstören, müssen die Neuen Staaten darauf bedacht sein, sie für sich und ihre Institutionen erst einmal zu begründen.

$*$

Hiermit ist der Rahmen in großen Linien abgesteckt, innerhalb dessen diese Untersuchung sich zu bewegen hat, und zugleich deren Gliederung vorgezeichnet. Als erstes wird demgemäß der Möglichkeit nachzugehen sein, daß die Neuen Staaten ihre ideologische Orientierung je für sich oder miteinander zu finden suchen (Teil A). Insofern sich ergeben wird, daß von dieser Möglichkeit nicht oder jedenfalls nicht ausschließlicher Gebrauch gemacht wird, muß gefragt werden, wo die Neuen Staaten eine Orientierung von dritter Seite suchen. Hierfür kommen in Betracht der „Westen“ (Teil B) und der „Osten“ (Teil C). In dem letzten Teil werden vor allem die "Sozialismen" der Neuen Staaten vorzuführen sein - und zwar immer unter dem Aspekt, ob und in welchem Maße es sich um Übernahmen aus dem "Osten“ handelt. Weil zu umfangreich, soll dieser letzte Themenkreis an anderer Stelle — in einem Beiheft dieser Zeitschrift - untersucht werden.

\section{A. Autarkie}

Staaten, die soeben ihre Unabhängigkeit errungen haben und sich ihrer deswegen noch nicht sicher fühlen, werden auf Abhängigkeiten aller Art sehr viel empfindlicher reagieren, als es alte, selbstsichere Staaten zu tun pflegen. Den Vorrang genießt infolgedessen das "Verlassen auf sich selbst“ - die "self-reliance“, jedenfalls mindestens als Ideal. Es kommt eine praktische Erwägung hinzu: Die Dritte Welt wünscht, sich nicht nur politisch, sondern vor allem auch ideologisch aus dem Gegensatz der beiden großen Weltlager herauszuhalten - hier wieder nicht allein um der Unabhängigkeit willen, sondern um in diese Auseinandersetzung nicht verstrickt $\mathrm{zu}$ werden ${ }^{17}$. Es handelt sich vor allem darum, den Eindruck zu vermeiden, daß eine Rezeption aus einem dieser Lager als Stellungnahme für dieses Lager oder gar als Zuordnung zu diesem mißdeutet werden könnte.

Den äußersten Fall von Autarkie bildet die Rückkehr zu vorkolonialen Zuständen und Auffassungen - also nicht nur der Verzicht auf Rezeption de futuro, sondern darüber hinaus die Ausmerzung allen dessen, was von den Kolonialherren ${ }^{18}$ übernommen worden war.

16 Vgl. hierzu Hector Monro, The Ambivalence of Bernard Mandeville (Clarendon Press, Oxford 1975).

17 Vgl. C. V. Crabb jr., The Elephants and the Grass - A Study of Nonalignment - (New York usw.

18 Zur Kolonialherrschaft vgl. jetzt R. von Albertini, Europäische Kolonialherrschaft $1880-1940$ (Zürich 1976). 


\section{Restauration der vorkolonialen Auffassungen und Zustände?}

Man kann ohne weiteres feststellen, daß keiner der Neuen Staaten sich für eine solche Restauration entschieden hat. Diese Entscheidung scheint mir nicht so selbstverständlich zu sein, wie man es offenbar überall annimmt: Ihre grundlegende Bedeutung sollte daher stark hervorgehoben werden. Diese Feststellung wird bestätigt dadurch, daß sich auch innerhalb dieser Staaten keinerlei Anzeichen dafür finden, $\mathrm{da}$ ein solcher Sprung zurück in die Vergangenheit angestrebt werde. Es gibt im ganzen wohl nur zwei zudem kleine Parteien, die dergleichen auf ihre Fahnen geschrieben haben: In Indien die Jana Sangh ${ }^{19}$ und in der Türkei die Heilspartei ${ }^{20}$. Gelegentlich scheinen, wie z. B. in Ägypten, Islamische Brüderschaften der Modernisierung - vergeblichen - Widerstand entgegengesetzt zu haben. Ob man in der Ausrufung des Kaisertums in Zentralafrika "einen Schritt zurück“ zu sehen hat, muß sich erst zeigen ${ }^{21}$. Das Gesamtbild zeigt eindeutig und eindrucksvoll das, was J. S. Coleman von Afrika feststellt: „In a word, futurism, not traditionalism, has been the ethos of African nationalism"22.

Den handgreiflichsten Beweis für eine solche Gesinnung hat Somalia geliefert, als es Männer hat erschießen lassen, die gegen die Emanzipation der Frau Stellung genommen hatten ${ }^{23}$. Demgegenüber kommen einzelne traditionalistische Regungen nicht in Betracht. Hierher gehört etwa die Umbenennung der neuen Staaten: Wenn man an ihrer von den Kolonialmächten allein nach ihren Interessen geschaffenen Gestalt nichts ändert, dann fällt die Ersetzung des alten durch einen neuen Namen - zuerst etwa von Congo in Zaire ${ }^{24}$, zuletzt etwa von Dahomey in Benin - oder die Verdrängung christlicher Vornamen durch afrikanische, wie dies etwa durch Mobutu geschehen ist ${ }^{25}$, schwerlich ins Gewicht ${ }^{26}$. Im übrigen kann man selbst bei Rückgriff auf die Vergangenheit nicht sicher sein, daß es sich wirklich um eigene und nicht doch wieder um rezipierte Vergangenheit handelt ${ }^{27} 28$.

Besonderer Hervorhebung bedarf in diesem Zusammenhang der Fall Kamerun ${ }^{29}$. Diese deutsche Kolonie war nach dem Ersten Weltkrieg in ein englisches und in ein französisches Mandat aufgeteilt worden. Nachdem diese beiden Teile die Unabhängigkeit erlangt hatten, entschieden sie sich nicht etwa für Selbständigkeit oder - der englische Teil - für den Anschluß an Nigeria ${ }^{30}$, sondern sprachen sich sehr energisch für "Wiedervereinigung" aus und verwirklichten diese zunächst in Gestalt eines Bundes-, dann als Einheitsstaat ${ }^{31}$. Man kann hierin den extremen 19 Vgl. Craig Baxter, Jana Sangh - An Biography of an Indian Political Party - (Pennsylvania U..P 1969),
etwa S. 149 und S. 160. Angela S. Burger, Opposition in a Dominant-Party-System (California U.P. 1969).

20 Vgl. „Frankfurter Allgemeine Zeitung“ (FAZ) vom 17. Mai 1976: „Der türkische Staat öffnet sich wieder dem Islam.“ Dazu auch Bulent Nuri Esen, La Turquie (Paris o. J.), S. $229 \mathrm{f}$.

21 Vgl. "Neue Zürcher Zeitung" vom 7 . Dezember 1976.

22 Vgl. "J. S. Coleman, Tradition and Nationalism in Tropical Africa, in Martin Kilson (ed.), New States in the Modern World (= Festschrift für Rupert Emerson), Harvard U.P. 1975, S. 3 ff., S. 3.

23 B. O. Bryde a. a. O. (oben Anm. 4), S. 176.

$24 \mathrm{Vgl}$. NZZ vom 29. Oktober 1971.

25 Vgl. Brun-Otto Bryde a. a. O., S. 134.

26 Von der Re-Islamisierung Libyens wird später die Rede sein.

27 Vgl. Benedict R. O. G. Anderson, The Idea of Power in Javanese culture, in: C. Holt (ed.), Culture and Politics in Indonesia (Cornell U.P. 1972), S. 1 ff., S. 5.

$28 \mathrm{Da}$ die Orientierung an der eigenen Geschichte auch zu verhängnisvollen Fehlern führen kann, zeigt sehr anschaulich Ernest R. May, "Lessons* of the Past (New York Oxfort U.P. 1973) an den Vereinigten Staaten: Dort ist man ohne weiteres davon ausgegangen, die Situationen des Zweiten Weltkrieges seien die gleichen wie die des Ersten und hat daher seinerzeit vermeintlich gemachte Fehler korrigiert, mit dem Ergebnis, daß diese Korrekturen in den gründlich veränderten Situationen des Zweiten Weltkrieges sich als ebenso verfehlt erwiesen haben wie die Entscheidungen im Ersten Weltkrieg.

$29 \mathrm{Vgl}$ : zum folgenden W. R. Johnson, The Cameroon Federation-Political Integration in a Fragmentary Society (Princeton U.P. 1970).

30 Vgl. zu der sich zur. Wiedervereinigung bekennenden Volksabstimmung W. Frhr. von Blittersdorff, Das Internationale Plebiszit (Hamburg 1965).

31 Vgl. NZZ vom 25. Mai 1972. 
Gegenfall von Restauration sehen: Das vorkoloniale Alte wird nicht nur nicht wiederhergestellt - was wiederhergestellt wird, ist genau das, was eine Restauration hätte beseitigen sollen.

Mit einer solchen Entscheidung haben es sich die Neuen Staaten selbst unmöglich gemacht, den Kolonialismus ausschließlich unter dem Blickwinkel von Schuld und Sühne zu betrachten: Sie haben durch diese Entscheidung zu erkennen gegeben, das Kolonialzeitalter als eine geschichtliche Begegnung zu sehen, mittels derer ein unbekannter und rätselhafter Urheber das Rad der Geschichte eine unerhörte Strecke weiterbewegt hat. Nicht übersehen werden darf in diesem Zusammenhang vor allem, daß die Berührung mit den Kolonialherren diejenige einheimische Elite hervorgebracht hat, die nicht nur zur Abschüttelung dieser Herrschaft notwendig war, die vielmehr auch der Entscheidung für die Modernität Inhalt geben konnte ${ }^{32}$. Diese Deutung wird bestätigt durch die Beobachtung, daß sich die Feindschaft der ehemaligen Kolonialvölker gegen ihre früheren Kolonialherren immer mehr abschwächt, ja weithin freundschaftlichen Beziehungen Platz gemacht hat ${ }^{33}$, die allerdings weniger auf der Fortführung alter als auf der Anknüpfung neuer Beziehungen $\mathrm{zu}$ beruhen scheinen ${ }^{34}$.

Es zeigt sich insgesamt, daß die Neuen Staaten sich geistig nicht nur nicht auf sich selbst zurückgezogen haben, daß sie sich vielmehr für die Kommunikation mit aller Welt einschließlich der früheren Herren offenhalten. Garanten und Medien einer solchen Beziehung zum Westen sind einstweilen noch vor allem die Großen Alten Männer der Dekolonisation und Staatsbildung - wie Bourgiba, Senghor, Houphouet-Boigny, Kenyatta u. $\mathbf{a} \mathbf{}^{35}$.

\section{Beschreiten eines „Dritten Weges“?}

Diese Alternative der geistigen Autarkie unterscheidet sich von der vorhergehenden dadurch, daß man zwar aus der eigenen geistigen Kraft zu leben hofft - im ersten Falle von dem, was die Nation bereits geleistet hat, im zweiten Falle jedoch auf neue Fruchtbarkeit setzt und hiermit die Probleme des nachkolonialen Zeitalters zu bewältigen sucht.

Eine solche Lösung muß einmal der „Dritten Welt" ungemein zusagen. Weltpolitisch sucht sie sich von einer Verstrickung in die Ost-West-Auseinandersetzung freizuhalten: Ihr Ideal ist bekannt unter den Titeln "nonalignment“ mit dem Ergebnis der Dritten Welt als „noncomitted area"36. Wir haben früher bereits angedeutet, daß die neuen Staaten dieserhalb Rezeptionen von Geistesgut aus einem

32 Vgl. hierzu die noch immer unübertroffene Schilderung durch J. S. Coleman, Nigeria - Background to Nationalism - (California U.P. 1958/1971). Vgl. hierzu auch den französischen Ministerpräsidenten Mendès-France 1954 in Tunis: „... La valeur remarquable de ses élites justifie que ce peuple soit Mendés-France 1954 in Tunis: ". La va valeur remarquable de ses élites justifie que ce peuple soit
appelé à gérer lui-même ses "affaires". Nach A. Nouschi, Histoire, Politique et Legitimité au Maghreb, in: M. Teitler u. a., Elites, Pouvoir et Legitimité au Maghreb (Paris 1973), S. 15 ff., S. 16.:

33 Moralische Entrüstung über den - westlichen - Kolonialismus gibt es nur noch im Osten. Hier geht es jedoch nicht um das. Wohl der neuen. Völker, sondern es handelt sich um eine Waffe der psychologischen Kriegführung - hier der Diskreditierung des Westens vor der Dritten Welt.

$34 \mathrm{Vgl}$. etwa für Zaire R. Cornevin, Le Zaïre (Paris 1972), S. 123 f. Über das Absterben der alten Beziehungen, die zwischen England und Indien bestanden hatten, vgl. Michael Lipton und John Firn, The Erosion of a Relationship - India and Britain since $1960-$ (Oxford U.P. 1975).

35 Hierzu noch Robert L. Hess, Italian Colonialism in Somalia (Chicago U.P. 1966), S. VII: \#. . the present Somalia Republic ... is the political descendant not of tribal Somalia, but of the Italian colony of Somalia as well as of its success of governements".

$36 \mathrm{Vgl}$. hierzu außer dem oben Anm. 17 genannten Werk noch: Peter Lyon, Neutralism (Leicester U.P. 1963), Peter Worsley. The Third World - A vital new force in international affairs - (Chicago U.P. 1965). 
dieser Lager mit Zurückhaltung gegenüberstehen, um auch nur den Eindruck zu vermeiden, sie stünden auf seiten einer dieser großen Parteien oder hätten sich gar einem Block angeschlossen. Es ist offenkundig, daß ein „Dritter Weg“ geeignet wäre, die Blockfreiheit der neuen Staaten auch ideologisch zu gewährleisten, ohne daß man deswegen auf veraltetes Gedankengut aus der vorkolonialen Zeit verwiesen wäre. Aber auch für Ost und West scheint eine solche frisch aufbrechende Fruchtbarkeit der Neuen Staaten erwünscht, wenn nicht sogar notwendig. Beide Blöcke haben sich nämlich nicht nur einer Ideologie verschrieben - sie haben ebenso diese Ideologie zu einer (Pseudo-)Religion gesteigert oder vielmehr denaturiert. Der Beweis für diese Beurteilung liegt vor allem in dem unbedingten „EntwederOder"37 dieser "Ideologien“. Wie es einer Religion ansteht, betrachten sich beide als letzte und endgültige Offenbarungen, mit zwei Folgen: Ist die eine „Religion“, wie es der Natur der Sache entspricht, von Gott und seinen Propheten verkündet, dann können alle anderen Ideologien nur des Teufels sein - es gibt keine Diskussion, keinen Kompromiß und erst recht keine Rezeption; zweitens kann es aber auch keine Anderung oder gar Vervollkommnung aus der Ideologie selbst heraus geben, wenn sie das letzte Wort Gottes ist - mit der Denaturierung der Ideologie zur Religion hat man sich auf beiden Seiten selbst zu Immobilismus und Sterilität verurteilt. Gelänge es den Neuen Staaten, einen Dritten Weg zu finden, der über beide erstarrten und unfruchtbaren Ideologien hinausführen würde, dann könnte sich dies als ein Anstoß zur ideologischen Selbstbefreiung von Ost und West auswirken - sie könnten sich vielleicht daraufhin ihrerseits veranlaßt fühlen, in ihrem Sinne einen Dritten Weg zu suchen.

1. Mit diesem Ansatz stellt sich als erstes die Frage, wie man die Aussichten für einen solchen Ausbruch von geistiger Fruchtbarkeit zu beurteilen hat. Geht man davon aus, daß eine solche Fruchtbarkeit die Gabe ist, mit der die Geschichte unerforschbar die Völker beschenkt, dann lassen sich Prognosen nicht stellen. Insbesondere ergibt sich daraus, daß sich solche Fruchtbarkeit anders als die Modernität nicht „machen" läßt. Aber immerhin scheint das Phänomen „Schicksal“ ein Sympton dafür zu sein, daß ein Aufbruch zu neuen Ufern in Betracht kommt mit der Folgerung, daß man jedenfalls dem Eintritt eines solchen Ereignisses nicht zuwiderarbeiten sollte, und zwar insbesondere nicht seitens des östlichen oder westlichen Lagers. Als „Schicksal“ ist für die Neuen Staaten insbesondere zu sehen die dramatische, ja tragische Begegnung mit der Alten Welt und der Kampf um die Selbständigkeit, der vollen Einsatz vor allem auch der geistigen Kräfte gefordert hat und sie damit in eine Bewegung versetzt haben sollte, deren Fruchtbarkeit sich nicht in dem Akt der Verselbständigung erschöpft haben muß, die vielmehr erst und vor allem nach diesem geschichtlichen Augenblick durch dessen Auswertung und Ausbildung sich zur Geltung bringen sollte. Wie bereits angedeutet, läßt man sich diese Chance der Schöpfung entgehen, wenn man die Ereignisse lediglich moralisch betrachtet und auf „Schadensersatz" plädiert: Hierdurch wird erlittenes Unrecht gerade nicht in Produktivität umgesetzt; denn Schadensersatz meint Naturalrestitution, also Wiederherstellung des Zustandes, wie er bestehen würde, wenn die

37 Dieser Charakter bestätigt sich durch die Tonart, in der von anderen, nicht einmal grundsätzlich gegenteiligen Meinungen gesprochen wird: Es handelt sich hier um nichts anderes als die altbekannten "Rabies theologica"; ein Beispiel hierfür bietet E.-J. Mestmäcker, Wirtschaftsordnung und Staatsverfassung, i. Festschrift für Franz Böhm (Tübingen 1975), S. $383 \mathrm{ff}$. In der Sache macht sich diese Wendung dadurch bemerkbar, daß die andere Meinung nicht mehr auf ihre Richtigkeit, sondern auf ihre Sittlichkeit beurteilt und der Andersdenkende nicht als Irrender, sondern als Sünder verurteilt wird. 
koloniale Begegnung nicht stattgefunden hätte - kurz gesagt also sterile Restauration statt fruchtbarer Revolution.

Diese Hypothese bedarf der Verifizierung. Beginnt man sie mit den einzelnen Staaten, dann wird man vor allem darauf $\mathrm{zu}$ achten haben, ob und wie sie die übernommenen politischen usw. Institutionen verändern. In der Praxis wird man in diesem Lichte vor allem zu prüfen haben „Einparteiensystem“ und „Militärregime": Muß man doch damit rechnen, daß es sich nicht um "Abweichungen" vom westlichen oder östlichen Ideal handelt, die man eben als solche zu verurteilen und zu bekämpfen hat, daß sich hier vielmehr die allerdings unvollkommenen, ja mißgestalteten Anfänge von „dritten“ Neubildungen zeigen könnten ${ }^{38}$.

Der Untersuchung bedarf zweitens die Möglichkeit, daß die Neuen Staaten unter sich in eine Kommunikation treten, durch die sie sich anregen und befruchten lassen. In dieser Hinsicht fehlt es gewiß nicht an wirtschaftlicher Entwicklungshilfe. $\mathrm{Zu}$ prüfen wäre also, ob es etwas Derartiges auch in ideologischer Hinsicht gibt. Eine solche Prüfung ergibt nichts für die großen Konferenzen der Dritten Welt, wie sie 1955 in Bandung ${ }^{39}$ begonnen und sich einstweilen bis Colombo 1976 fortgesetzt haben. Folgt man den Zeitungen, dann ist in Sri Lanka nicht mehr herausgekommen als scharfe und schärfste Kritik an der Alten Welt und ihrem alten und neuen Kolonialismus.

In diesem Rahmen muß man ferner der Möglichkeit nachgehen, daß innerhalb der Dritten Welt Gedankengebäude eine „Renaissance“ erleben und daß die Neuen Staaten hiernach greifen. Hier ist an erster Stelle des Islam ${ }^{40}$ und des Arabismus zu gedenken. Der Zweifel, ob der Islam dem Modernismus feindlich oder wenigstens neutral gegenübersteht, dürfte zugunsten der zweiten Alternative entschieden sein. Zuletzt hat Maxime Rodinson ${ }^{41}$ in eingehender Darstellung zweierlei nachzuweisen gesucht: Einmal, daß das heilige Recht, die Sharia, keineswegs unveränderlich sei, d. h. also neuen Bildungen nicht entgegenstehe; und daß der Islam alles andere als den Kapitalismus verdamme, ganz abgesehen davon, daß man für diesen Fall hervorragende Techniken der Umgehung seiner Gebote und Verbote entwikkelt habe. Vor allem ist keineswegs ausgemacht, daß der Islam den Fatalismus predige: Bedenkt man, daß er letzte Aktivität in der Verbreitung des Glaubens in Gestalt des Heiligen Krieges verlangt, dann mag eine Haltung, die in dieser Hinsicht obsolet geworden ist, sich um so mehr verweltlicht als politische, ökonomische usw. Aktivität äußern.

Ein Blick auf den Arabismus bestätigt dies alles. Er hat sich nach dem Kriege in der Errichtung der Arabischen Liga 194542, in der Begründung eines Gemeinsamen Marktes $^{43}$ und in lebhafter außenpolitischer Rührigkeit geäußert ${ }^{44}$. Ideologisch ist in der Gruppe immer wieder von „Sozialismus“ die Rede. Damit stellen sich

38 Vgl. Herbert Krüger, Militärregime in Übersee 1976 - Beiheft 8 dieser Zeitschrift.

39 Vgl. NZZ vom 1. April 1976 ,Jugoslawien als Wortführer der Blockfreien“; vom 15. April 1976: „Brüchiger Block der. Blockfreien“; und vom 18. April 1976: „Tag der Scharfmacher unter den Blockfreien“. Hierzu Dieter Schröder, Die Konferenzen der "Dritten Welt “ (Hamburg 1968).

40 Der Verfasser stützt sich hier vor allem auf Louis Gardet, La Cité Musulmane (Paris 1969), und W. W. Watt, Islamic Political Thought (Edinburgh U.P. 1968).

41 Islam and Capitalism (New York o. J.). Es handelt sich um die Übersetzung eines 1966 unter dem Titel "Islam et Capitalism" in den Editions du Seuil erschienenen Werkes. Michael Brett (ed.), Northern Africa - Islam and Modernization - (London 1973) enthält allerdings von dem Untertitel am wenigsten.

42 Vgl. R. W. Mac Donald, The League of the Arab States (Princeton U.P. 1965)

43 Vgl. Alfred G. Musrey, An Arab Common Market - A Study in inter-Arab Trade Relations, $1920-67$ (New York 1969).

44 Und zwar auch abgesehen von Israel; hierzu etwa H. A. Hassouna, The League of Arab States and Regional Disputes (Oceana Publications 1975) - Eine Besprechung findet sich im AJIL 76, S. 613. 
zwei große Probleme: Einmal muß ermittelt werden, ob dieser Sozialismus wirklich ein Eigengewächs ist, oder ob es sich um eine mehr oder weniger verhüllte Übernahme aus dem Osten handelt. Dazu bedarf es insbesondere der Klärung - Eigenständigkeit unterstellt —, wie sich dieser Sozialismus zu den mannigfachen asiatischen, afrikanischen und lateinamerikanischen Sozialismen verhält, ob es sich also um eigentümliche Hervorbringungen handelt, oder ob ein Austausch stattgefunden hat und ob sich schließlich alle individuellen Bildungen dieser Art zu einem asiatischen, afrikanischen, lateinamerikanischen oder gar zu einem „Sozialismus der Dritten Welt" vereinigt haben. Zweitens ist hiermit zugleich zu untersuchen, ob diese Sozialismen oder dieser Sozialismus ein Symptom für eine geistige Fruchtbarkeit sind, die unter dem Stern der Herausforderung steht, die die Erringung einer eigenen staatlichen Existenz darstellt ${ }^{45}$.

Macht man die Probe aufs Exempel ${ }^{46}$, dann scheint es mindestens zwei Felder zu geben, auf denen die Neuen Staaten eine ebenso eigenartige wie beachtliche geistige Fruchtbarkeit zeigen. Es handelt sich einmal um das Offentliche Seerecht. Hier ist es insbesondere die Konzeption der "Wirtschaftszone“, die sie gefunden und so gut wie durchgesetzt haben: Man muß geradezu sagen, daß sie mit der Erfindung dieses Institutes die westliche und östliche Welt in die Defensive gedrängt haben, um von deren wirtschaftlichen Einbußen ganz zu schweigen. Das zweite Feld ist die Weltwirtschaftsverfassung ${ }^{47}$. Man kann nicht leugnen, daß den Neuen Staaten hierzu Entscheidendes eingefallen ist, gleich ob es einem paßt oder nicht, und daß sie auch an dieser Stelle das Gesetz des Handelns in die Hand genommen haben: Es genügt, an die Konferenz von Nairobi zu erinnern ${ }^{48}$.

2. Die den Neuen Staaten zufallende geschichtliche Aufgabe, neue "dritte Ideen“ in die internationale Diskussion, insbesondere von Zukunftsproblemen, einzuführen, setzt eine Prüfung der hierfür in Betracht kommenden Begriffe, Formeln usw. voraus, ob es sich wirklich um Gedanken handelt und ob gegebenenfalls diese Gedanken ursprüngliche und wahrhaft neue sind.

Mit der ersten Möglichkeit, daß es sich nämlich eher um Worte als um Ideen handelt, wird man vor allem zu rechnen haben, wenn von "Sozialismus" die Rede ist. Man mag diese Parole einmal gewählt haben um der bloßen Verneinung und Absetzung von Kapitalismus, Imperialismus und Kolonialismus willen: Will man sich von alledem entschieden distanzieren und vor allem auch eine solche Distanzierung handgreiflich bekunden, dann kommt hierfür nur die absolute Gegenposition, eben "Sozialismus“ in Betracht ${ }^{49}$. Eine andere Variante eines Lippenbekennt-

$45 \mathrm{Vgl}$. hierzu unten. Nicht übersehen werden darf, daß gerade der Stolz auf diese Existenz als Ursache eines Verzichts auf Übernahme wirken kann. Ein Beispiel hierfür bietet Alexander B. Woodside in seinem vortrefflichen Werk "Community and Revolution in Modern Vietnam “ (Boston Mass 1976), S. 251: "Supporters of the more extreme Chinese example of agricultural change existed with the Vietnamese movement, but they were rebuked by the December 1963 meeting of the executive committee of the Vietnamese Party Central for lacking a spirit of independence" and for not daring to wash away the mentality of servitude to foreign countries produced by the residue of influence of been ruled for a thousand years (by China) “

46 Soweit ersichtlich, gibt es nur einen Versuch, die Möglichkeit eines „Dritten Weges“ wissenschaftlich zu untersuchen: Emile-Désiré's Dissertation „Der Traum einer Dritten Welt “ mit dem Untertitel „Quellen und Bedeutung des afrikanischen Sozialismus“ (Köln 1966).

47 Vgl. Christian Tomuschat, Die Charta der wirtschaftlichen Rechte und Pflichten der Staaten, in: Zeitschrift für Ausländisches Offentliches Recht und Völkerrecht 36 (1976), S. $444 \mathrm{ff}$.

$48 \mathrm{Im}$ Völkerrecht überhaupt und in der auswärtigen Politik läßt sich Entsprechendes allenfalls in Anzeichen oder vor allem Vorbehalten gegenüber den klassischen europäischen Lösungen erkennen; vgl. für das Völkerrecht Walter Rudolf, Neue Staaten und das Völkerrecht, in: Archiv des Völkerrechts 17 (Tübingen 1976), S. $1 \mathrm{ff}$;; vgl. für die Außenpolitik etwa Michael Leifer, The Foreign Relations of the New States (Camberwell Australia 1974) und Baghat Korany. Social Change, Charisma and Inter national Behaviour: Toward a Theory of Foreign Policy-making in the Third World - (Leiden 1976). (Syracuse U.P. 1966), S. $321 \mathrm{ff}$.

49 Vgl. J. C. Livingston and R. G. Thompson, The Consent of the Governed, 2. Aufl. (New York usw. 1966), S. 48, sie weisen mit Recht darauf hin, daß „for most of the uncommitted peoples of the world, 
nisses zum Sozialismus mag mit Daniel E. Apter ${ }^{50}$ darauf zurückzuführen sein, daß diese Ideologie (scheinbar!) alles ungemein einfach erklärt und ihre Verheißungen ebenso ungemein leicht zu verwirklichen sind. Es bedarf keiner Darlegung, daß es sich in diesen und ähnlichen Fällen nicht um jene Art von Hervorbringungen handelt, die als Ideen bezeichnet werden können.

Als sehr viel schwieriger erweist sich die Klärung der zweiten Frage, ob echte Ideen in der Substanz ursprünglich und neu sind, oder ob hier nicht lediglich alter Wein in neue Schläuche gefüllt worden ist. Diese Möglichkeit hätte lediglich dann von vornherein auszuscheiden, wenn sich ein Staat nach außen völlig und erfolgreich abgeschlossen hätte. Die kommunistischen Staaten haben sich zwar eine solche Abschließung vorgenommen, aber die Entwicklung der Technik hat einen Strich durch diese Rechnung gemacht: Für die modernen Kommunikationsmittel gibt es keine undurchdringlichen Räume mehr. Tritt daher heute in einem Staate eine frische Überlegung hervor, dann muß in jedem Falle damit gerechnet werden, daß fremde Zeugung oder auswärtiger Anstoß hier am Werke gewesen ist. Es wäre jedoch voreilig, hieraus den Schluß zu ziehen, es gebe allenthalben nur noch rezipiertes Geistesgut. Man muß nämlich bedenken, daß es eine vollständige und unbedingte Ursprünglichkeit niemals gegeben hat, wie etwa die Geschichte der Sprachen zeigt. Man hat daher eine solche Qualifikation zu relativieren und darauf abzuheben, ob aus eigenen und fremden Elementen etwas Drittes geschaffen worden ist. Wenn somit die technische Perfektion der Kommunikation Ursprünglichkeit nicht ausschließt, ja vielleicht sogar begünstigt, vorausgesetzt, daß ein schöpferischer Genius am Werke ist, dann ist damit zugleich der weitere Gang unserer Untersuchung vorgezeichnet: Wenn ein in der Dritten Welt auftretender Gedanke oder seine Bezeichnung identisch oder ähnlich sind mit entsprechenden Begriffen, Instituten usw. des Westens oder des Ostens, dann läßt sich die Alternative zwischen Rezeption und Parallele nur klären, indem man beide Phänomene miteinander konfrontiert und ihren Inhalt miteinander vergleicht. Die zeitliche Priorität begründet dann die Vermutung einer Übertragung, die jedoch der Widerlegung zugänglich ist.

3. Schließlich muß die Frage aufgeworfen werden, ob die Hervorbringung wesentlicher eigener Ideen von spezifischen "Geburtshelfern" unterstützt und gefördert wird. Die Übernahme und Anerkennung einer solchen Rolle setzt zweierlei voraus: Einmal darf ein solcher Helfer nicht eine Weltmacht sein: Sogleich nämlich würde sie dann einen Nebengeschmack nach „Neo-Kolonilismus“ annehmen. Zweitens mag der Geber durchaus einem der beiden Blöcke angehören. Aber das, was er geistig zu bieten hat, muß doch gegenüber der kommunen Ideologie des Blockes so bedeutende Eigenheiten aufweisen, daß eine Beschäftigung damit nicht als eine Identifizierung qua Ideologie mit dem in Frage stehenden Block mißdeutet werden kann und daß selbst dann, wenn der dritte Staat dieser Ideologie nichts abzugewinnen vermag, dies doch nicht für die eigenartigen Gestaltungen des „Geburtshelfers" zu gelten braucht, diese vielmehr durchaus ansprechen können.

Im Westen ist es Frankreich, im Osten ist es Jugoslawien, die für eine solche Rolle in Betracht kommen.

capitalism is, for valid historical reasons, identified with colonialism and white supremacy". Woodside a. a. O. (oben Anm. 1), S. 94, macht treffend darauf aufmerksam, daß diese Sicht nicht autochthon, sondern aus dem Westen entlehnt ist.

50 The Politics of Modernization (Chicago U.P. 1965), S. 321/322. 
a) Es scheint, als ob General de Gaulle an eine solche Rolle für sich und für Frankreich gedacht hätte ${ }^{51}$. Eine solche Hegemonie des Esprit hätte Frankreichs Stellung in der Welt nicht unverächtlich gesteigert, worauf es dem General gewiß nicht zuletzt angekommen ist, zumal auf diese Weise das Mißverhältnis zwischen hohen weltpolitischen Ansprüchen und natürlichen Ressourcen gemindert worden wäre. Beide Voraussetzungen für eine solche Rolle sind gegeben: Innerhalb des westlichen Lagers wird von Frankreich sehr entschieden ein erhebliches Maß von Selbständigkeit behauptet. Ferner verfügt Frankreich in der Verfassung von 1958 über eine Hervorbringung, die über den üblichen Typ westlicher Verfassungen wesentlich hinausführt, und zwar in einer Richtung, wie sie auch den Staaten der Dritten Welt vorschwebt: Für die Verfolgung von „Modernisierung“ bedürfen sie in erster Linie einer handlungs- und leistungsfähigen Regierung, und dies ist der Punkt, auf den es der Verfassung von 1958 entscheidend ankommt und in dem sie sogar so weit geht, daß sie der Regierung ein "Selbständiges Verordnungsrecht" dadurch zuspricht, daß sie die Gesetzgebungszuständigkeit des Parlamentes auf bestimmte, aufgezählte Gegenstände beschränkt. Es nimmt daher die Zahl der Verfassungen nicht wunder, die sich an diesem Vorbild orientiert haben ${ }^{52}$.

b) Was im Westen Frankreichs für die Dritte Welt bedeutet, mag im Osten von Jugoslawien ${ }^{53}$ gelten. Einmal gehört es organisatorisch nicht dem Ostblock an, und was die kommunistische Ideologie angeht, so hat dieses Land ihr eine besondere Note durch den Gedanken der "Selbstverwaltung der Unternehmen" verliehen ${ }^{54}$. Die auf solchen Voraussetzungen beruhenden Wirkungen haben sich eingestellt. Einmal betrachtet die Dritte Welt Marschall Tito als einen, wenn nicht sogar den hervorragendsten ihrer Sprecher ${ }^{55}$, wodurch Jugoslawien einen Rückhalt erhält, über den es von Hause aus nicht verfügt. Ferner hat die Figur "Selbstverwaltung“ eine gewisse Aufmerksamkeit erregt: Man hat insbesondere die "autogestion“ der algerischen Staatsgüter durch die Arbeiter hiermit in Verbindung gebracht ${ }^{56}$.

Hiermit sei fürs erste der Versuch abgebrochen, eine Vorstellung davon zu gewinnen, ob die Neuen Staaten aus sich heraus entweder je für sich oder miteinander $\mathrm{zu}$ bereits faßlichen schöpferischen Hervorbringungen gelangt sind. Die Themen, denen Abschnitt $C$ gewidmet ist, unter ihnen insbesondere die „Sozialismen“, werden Gelegenheit bieten, diesen Versuch wiederaufzunehmen.

\footnotetext{
51 Vgl. hierzu W. W. Kulski, De Gaulle and the World - The Foreign Policy of the Fifth Republic Syracuse U.P. 1966), S. $321 \mathrm{ff}$.

52 Vgl. hierzu Enno Kliesch, Einfluß des französischen Verfassungsdenkens auf afrikanische Verfassungen (Hamburg 1967). Georges Burdeau, Traité de Science Politique IV (2. Aufl. Paris 1969), S. 242. Eugen Fehr, Demokratische Staatsformen in Westafrika (Zürich 1965), S. 70, 132; Guy de Lusignan, FrenchSpeaking Africa since Independence (London 1969), S. 75. Edward J. Schumacher, Politics, Bureaucracy, and Rural Development in Senegal (California U.P. 1975), S. 63, 69, 72. Aristide R. Zolberg, One-Party Government in the Ivory Coast (Princeton U.P. 1969), S. 252. Zu diesen Regelungen der Verfassung 1958 vgl. Pierre Avril, Le Régime Politique de la Ve République (Paris 1967), S. 29.

53 Zur Verfassung dieses Landes vgl. zuletzt etwa Fred Singleton, Twentieth Century Yugoslavia (Columbia U.P. 1976), S. 150 ff., 225.

54 Zur Selbstverwaltung vgl. Herbert Krüger, Paritätische Mitbestimmung - Unternehmensverfassung Mitbestimmung der Allgemeinheit (Düsseldorf 1973), S. 28, mit weiterer Literatur. Ferner noch H.-C. Reichel, Die neue Jugoslawische Bundesverfassung, in: Osteuropa Recht 1974, S. 165 ff., und Christine Weyand, Das Delegiertensystem in der SFR Jugoslawien, ebenda 1976, S. $29 \mathrm{ff}$.

55 Vgl. NZZ oben und dieselbe schon vom 1. Febr. 1970: Titos persönlicher Einsatz für den Neutralismus. 56 Herbert Krüger a. a. O.
} 


\section{B. Intellektuelle Kommunikation zwischen dem „Westen“ und den Neuen Staaten}

Man kann schwerlich sagen, daß die zwischen den Völkern verschiedener Kontinente geknüpften und bestehenden geistigen Beziehungen sich eines Übermaßes an wissenschaftlicher Ergründung zu erfreuen hätten: Es steht hier nicht anders als mit der Überseeischen Verfassungsvergleichung. Zwar wird ausgiebig behandelt, was der Westen fremden Erdteilen an Menschen, Kapital ${ }^{57}$ und Technik geliefert hat. Aber daß daneben nicht nur eine Übertragung von Religionen, insbesondere des Christentums ${ }^{58}$, sondern auch von Ideologien stattgefunden hat, läßt sich nur aus gelegentlichen Bemerkungen erschließen.

Das gilt insbesondere für die Frage, welche staats- und gesellschaftwissenschaftlichen Autoren ${ }^{59}$ über Europa hinaus ihren Einfluß auf Übersee ausgedehnt haben. Von John Locke allerdings steht fest, daß er die Auffassungen der Gründungsväter der Vereinigten Staaten entscheidend bestimmt hat ${ }^{60}$. Heute gehört es zu den Ironien der Geistesgeschichte, daß zwar Karl Marx eine weltweite Anerkennung vielleicht weniger als Wissenschaftler denn als Ideologe genießt, daß aber nicht der Westen, sondern der Osten ihn für sich in Anspruch nimmt, weil er in ihm den Schöpfer seiner Weltanschauung ehrt.

Unter solchen Umständen kann dieses Thema jedenfalls an dieser Stelle lediglich als Merkposten oder gar nur als Fehlanzeige behandelt werden ${ }^{61}$. Hier sind allenfalls zwei Vermutungen zulässig. Es handelt sich einmal um die Bedeutung Auguste Comte's für Lateinamerika ${ }^{62}$. In dem diesem Teil der Welt gewidmeten Schrifttum scheint mir unter diesem Vorzeichen sein Name verhältnismäßig am häufigsten vorzukommen ${ }^{63}$. Für die Wahrscheinlichkeit dieser Vermutung spricht die folgende Überlegung: Soweit das Bürgertum dieser Länder im Sinne der Zeit „liberal“ und insbesondere freigeistig ist, hat es sich dem Klerikalismus entgegenzustellen, und hierfür bietet die Philosophie Comte's mit ihrer Verneinung von Religion und Metaphysik und ihrer alleinigen Bejahung des Positivismus die geeignete geistige Grundlage. Wie tief sich diese Philosophie muß haben einwurzeln können, zeigt die Tatsache, welche Schwierigkeiten in Venezuela die Schaffung eines katholischen Bildungswesens zu überwinden hatte ${ }^{64} \mathbf{6 5}^{6}$.

Als zweiten darf in diesem Zusammenhang auf Jeremias Bentham ${ }^{66}$ hingewiesen werden - einen Autor, der — wie mir scheint - überhaupt in seiner Bedeutung

57 Hierzu Herbert Feis, Europe The World's Banker 1870-1914 (Yale U.P. 1930).

58 Von ihm und seiner Mission soll hier nicht die Rede sein.

59 Dichter, Schriftsteller u. ä. m. interessieren daher an dieser Stelle nicht, obwohl ihre Verbreitung über die Welt wahrscheinlich eine bedeutendere gewesen ist als die von Theoretikern; so berichtet z. B. Woodside a. a. O., man habe in Vietnam Dumas' Grafen von Monte Christo und Sherlock Holmes Kriminalromane gelesen.

$60 \mathrm{Vgl}$. etwa A. J. Beitzinger, A History of American Political Thought (New York und Toronto 1972)

61 Die Biographien großer Staats- und Gesellschaftsdenker pflegen deren Ausstrahlung in andere Länder und Erdteile nicht zu behandeln; eine Ausnahme für den Grafen Saint Simon; vgl. Rouchdi Fakkar. Sociologie, Socialisme et Internationalisme prémarxistes (Neuchâtel 1968).

$62 \mathrm{Vgl}$. T. Lynn Smith, The Influence of Auguste Comte ..., in: Brazilian Society (University of New Mexico Press o. J.), S. 9 ff.

63 Vgl. Samel P. Huntington, Political Order in Changing Societies (Yale U.P. 1968/1969), S. 203. Herbert S. Klein, Parties and Political Change in Bolivia 1880-1952 (Cambridge U.P. 1969), S. 19, $36,126$. Vincent Padgett, The Mexican Political System (Boston Mass. 1976), S. 21. R. E. Poppino, Brazil The Land and the People - (Oxford U.P. New York 1966), S. 201. Vgl. zu diesem Thema überhaupt H. E. Davis, Latin American Thought: A Historical Introduction (New York 1972).

64 Hierzu Daniel H. Levine. Conflict and Political Change in Venezuela (Princeton U.P. 1973).

65 Nach Hans-Ulrich Wehler, Der Aufstieg des Amerikanischen Imperialismus (Göttingen 1974), S. 110, hat sich der chilenische Präsident Balmacede von Gedanken des deutschen Volkswirtschaftlers Friedrich List beeinflussen lassen.

66 Vgl. hierzu Wilfried Löwenhaupt, Politischer Utilitarimus und bürgerliches Rechtsdenken (Berlin 1972). Zwar ist diese Schrift John Austion und seiner „Philosophie des positiven Rechts“ gewidmet, beschäftigt sich aber ausgiebig mit J. B. Ferner Mary P. Mack, Jeremy Bentham - A Odyssey of Ideas $1748-1792$ (London 1962). Dort wird S. 394 ff. J. B.s Interesse für Indien hervorgehoben. 
für die Entwicklung des modernen Wohlfahrtsstaates noch nicht voll gewürdigt worden ist. Dasselbe gilt erst recht natürlich für seine Wirkung in Übersee: Er scheint vor allem in Indien Anhänger gefunden zu haben ${ }^{67}$.

Der Verfasser wüßte nicht, welchen dem 20. Jahrhundert angehörenden Autor er in gleicher Weise herausheben sollte. Ein insbesondere ökonomischer Neo-Liberalismus ist für die neuen Staaten uninteressant, weil es ihnen darauf ankommen muß, nicht-ökonomischen Notwendigkeiten von vornherein in der Ökonomie Rechnung zu tragen - also sich die Mißlichkeit zu ersparen, erst nachträglich den Sozialstaat gegen die Vernachlässigung sozialer usw. Erwägungen durch eine hybride Wirtschaft mobilisieren zu müssen. Der westliche Neo-Marxismus hat zwar über Intellektuelle, die im Ausland, und zwar vor allem in Paris, studiert haben, Eingang gefunden ${ }^{68}$. Er hat sich jedoch in den sechziger Jahren unseres Jahrhunderts in eine ziemlich fade ${ }^{69}$ utopische Schwärmerei aufgelöst - bietet also etwas, was den Neuen Staaten am allerwenigsten von Nutzen sein kann.

Angesichts eines solchen Befundes stellt sich in der Tat die Frage, wer die Auffassungen des Westens in den neuen Staaten vertritt, insbesondere also ob es dort westlich orientierte Parteien gibt ${ }^{70}$. Offenbar fällt die Antwort auf diese Frage nicht befriedigend aus. Denn St. Hoffmann zieht (194) den Schluß: „What is at stake is how to promote, in the non-Communist world, the values on which most Americans agree". Etwas Derartiges ist offenbar nicht allein für die Vereinigten Staaten wichtig und notwendig: Jede Außenpolitik ist von vornherein zum Scheitern verurteilt, wenn die Partner einander unbekannt geblieben sind, weil sie sich nicht gegenseitig vor- und dargestellt haben ${ }^{71}$. Es ist also allein der Modus, der hier Probleme aufgibt. Die Grundfrage stellt sich dahin: Sollen sich die Neuen Staaten an der Alten Welt orientieren, oder sollen sie von der Alten Welt orientiert werden. Ausgeschlossen sein muß gewiß jeder Druck, der hinter die schlichte Darbietung gesetzt wird. Damit gerät mancherlei, was in der Praxis geschieht, ins Zwielicht. Es scheint allerdings schwer, von einer westlichen Welt, deren geistige Atmosphäre von einer Moralhaftigkeit beherrscht wird, die mir mit echter Sittlichkeit wenig und dafür um so mehr mit Erbaulichkeit, Koketterie und Scheinheiligkeit zu tun hat, zu erwarten, daß sie nicht jede Gelegenheit zur Missionierung ihrer Ideale nutzte. So bepackt man etwa Entwicklungshilfe mit Auflagen, die nicht allein die bestmögliche Verwendung sichern, sondern moralische Fortschritte bewirken sollen ${ }^{72}$, wenn sie nicht etwa "Verächtern von Menschenrechten" und hier insbesondere wieder Militärregimen von vornherein verweigert wird ${ }^{\mathbf{3}}$. Auch Militärhilfe wird in den Dienst solcher Propaganda der Tugend gestellt, insbesondere auch vorenthalten, wenn der Empfänger, mag er auch außenpolitisch noch so wichtig

67 Vgl. K. M. Panikkar, The Foundation of New India (London 1963), S. 26: „Bentham and the Utilitarians in England, which was destined to have so powerful an influence in the development of ideas in India ....

$68 \mathrm{Vgl}$. Charles F. Andrain, Democracy and Socialism: Ideologies of African Leaders, in: D. A. Apter (ed.), Ideology and Discontent (New York 1964), S. 155 ff., S. 156: "The ideology of Marx and Lenin has become part of the African intellectual heritage“.

69 Der Verf. denkt hierbei vor allem an Herbert Marcuses Rede vor kalifornischen Frauen.

70 Stanley Hoffmann, The State of War (New York usw. 1965), S. 172.

$71 \mathrm{Es}$ ist dies der vornehmste Sinn der auswärtigen Kulturpolitik; vgl. hierzu etwa Suzanne Balous, l'action culturelle de la france dans le monde (Paris 1970), und Gilbert Caty, La Coopération scientifique entre la France et le Tiers-Monde (Paris 1967).

72 Ein Beispiel hierfür findet sich bei Gregory Henderson, Korea - The Politics of the Vortex (Harvard U.P. 1968), S. 186.

73 Vgl. NZZ vom 4. Januar 1977: „Umstrittene USA-Militärhilfe - Sechs Länder trotz Mißachtung der Menschenrechte beliefert - Vor allem: FAZ vom 4. 1. 1977. 
sein, unter der Beschuldigung steht, sich völkerrechtswidrig verhalten zu haben ${ }^{74}$. Selbst Handelsverträgen ist eine solche Nutzbarmachung nicht erspart geblieben: Es sei an den nordamerikanischen-sow jetrussischen Handelsvertrag erinnert ${ }^{75}$. Es ist nicht allein die Erwägung, daß Religion und politische Sittlichkeit nicht durch Druck gefördert werden sollten, sondern auch die pragmatische Überlegung, daß man mit einer solchen Strategie genau das Gegenteil des Erstrebten bewirkt, die zu einem Verzicht auf diese Art von Stärkung der ideologischen Stellung des Westens in den Neuen Staaten zwingen ${ }^{75 a}$.

Vielleicht kann man die Abwesenheit des Westens mit seinen Ideologien in der Dritten Welt darauf zurückführen, daß seine Staaten sich dem „Prinzip der NichtIdentifikation" verschrieben, also sich die In-Eins-Setzung mit einer Ideologie versagt haben, damit ein Pluralismus auch in dieser Hinsicht freien Raum findet. Aber selbst wenn dies richtig wäre, dann gäbe es immer noch seine staatlichen usw. Institutionen, die den Neuen Staaten für eine Orientierung zu Gebote stünden. Es scheint denn auch so, als ob die ideologische Präsenz des Westens in der Dritten Welt weniger auf Ideologien als auf Institutionen beruhte, während für den Osten das Gegenteil zu gelten scheint. Diese Vermutung veranlaßt, von einer Betrachtung der Ideologien zu einer Würdigung der Institutionen, Formen usw. überzugehen.

1. Wir beginnen diesen tour d'horizon mit der vielleicht bemerkenswertesten Leistung des Westens, dem Modernen Staat. Es gibt keine dekolonisierte Nation, die sich nicht entschlossen hätte, als Gestalt ihrer politischen Organisation den Modernen Staat zu wählen. Diese Wahl beruht nicht allein auf dem - zufälligen - Umstand, daß sie kein anderes Bild vor Augen hatten als die Staatlichkeit ihrer Kolonialherren und sich daher mindestens zunächst einmal hieran orientiert hätten. Maßgebend ist vielmehr die Sachnotwendigkeit gewesen: Wenn man sich für „Modernisierung“ entscheidet, dann ist damit von der politischen Organisation ein Höchstmaß von Handlungs- und Leistungsfähigkeit verlangt, beginnend bereits mit der Kraft zur Zurückdrängung der traditionellen Strukturen. In dieser Hinsicht ist der Moderne Staat bisher durch nichts anderes übertroffen worden, wie nicht zuletzt seine Überwindung des Feudalismus in Europa bezeugt.

Es ist nicht notwendig, im einzelnen darzulegen, worin die Eigenart des Modernen Staates besteht und worauf seine Handlungs- und Leistungsfähigkeit beruht ${ }^{76}$. Hier ist lediglich hervorzuheben, daß der Staat eine Gestalt der Allgemeinheit ist, daß also alle Staatsangehörigen an ihm teilhaben und teilnehmen. Ausgeschlossen ist damit die Beschränkung der Staatlichkeit auf einen Teil der Nation, eine Besonderheit im allgemeinen („Proletarier") und einer Elite im besonderen („Staat als Instrument der Herrschaft der Kapitalisten über die Proletarier“). Wenn sich daher eine neue Nation für die Gestalt des Modernen Staates entscheidet, dann entscheidet sie sich damit zwangsläufig zugleich für "Allgemeinheit des Staatsbürgertums“. $\mathrm{Ob}$ aber einer solchen Konsequenz eine adäquate Wirklichkeit entspricht, das scheint die große Frage zu sein. Für eine Theorie der Rezeption bedeutet dies einen

\footnotetext{
74 Seit dem 5. Februar 1975 besteht ein USA-Waffenembargo gegenüber der Türkei wegen der Besetzung eines Teiles von Zypern.

H. E. Davis, Latin American Thought; A Historical Introduction (New York 1972).

$75 \mathrm{Er}$ war beiderseits paraphiert. Als der USA-Kongreß über die Ratifizierung beriet, setzte er Auflagen hinein, denen zufolge die USA aus dem Vertrag nur verpflichtet sein sollten, wenn die Sowjetunion die Ausreise jüdischer Staatsbürger gestatte. Man kann sich kaum wundern, wenn die Sowjetunion daraufhin vom Vertrag zurückgetreten ist.

75a Alle diese UUberlegungen haben seit dem Amtsantritt des Präsidenten Carter eine geradezu exemplarische Bestätigung gefunden, insbesondere auch, was die Reaktion der einer solchen Pädagogik unterzogenen lateinamerikanischen Staaten angeht.

76 Hierzu darf auf Herbert Krüger, Allgemeine Staatslehre (Stuttgart, 2, Aufl. 1966) verwiesen werden.
} 
Typ, der zwar eine fremde Institution übernimmt, sie aber lediglich als Fassade vor anderen Bildungen aufbaut, die alt oder neu oder vor allem neues Altes sein können.

Verfassungstheoretisch handelt es sich um das Problem der „Realen Machtfaktoren “. Zwar bestimmt die Verfassung, wer die Herrschaft innehaben soll - in der Verfassung eines Modernen Staates kann dies nur die Allgemeinheit der Staatsbürger sein. In Wirklichkeit ist es nur allzu oft eine Minderheit, die herrscht. Mit der praktischen Depossedierung der Allgemeinheit verbindet sich durchweg eine Veränderung des Herrschaftssinnes: Vermag eine Allgemeinheit geradezu per definitionem nur zum gemeinen Wohl zu herrschen, so hat eine Minderheit mindestens die Vermutung gegen sich, daß sie zu ihrem besonderen Wohle herrsche. Ein Blick auf die Neuen Staaten läßt befürchten, daß man zwar überall behauptet, Moderner Staat zu sein, daß man aber dieses Modell verbildet hat, indem eine Minderheit oder eine Elite an Stelle der Allgemeinheit mindestens de facto herrscht.

Immer wieder stößt man in der Literatur auf die Beobachtung, daß die Dekolonialisierung nicht Staatlichkeit und damit notwendigerweise Demokratie mit sich gebracht habe, daß vielmehr lediglich neue einheimische Herren sich an die Stelle der alten fremden Herren gesetzt hätten ${ }^{77}$, daß ferner der Titel Herrschaft der Kolonialzeit unverändert weitergeführt werde und daß man sich, obwohl Inländer, nichtsdestoweniger die Auslandsgehälter der Weißen weiterbewilligt habe.

Es mag möglich sein, daß hier die alte Vorstellung nachwirkt, daß sich Herrschaft auf Eroberung gründet und sich die Ausbeutung der Beherrschten durch die Herrscher hierdurch legitimiere ${ }^{78}$. Wahrscheinlicher ist, daß es sich um eine von modernen Managern bewirkte Neuauflage jener militärischen oder bürokratischen Kasten handelt, die Land und Leute sls ihr Patrimonium betrachten, unter Ausschluß allerdings einer Fürsorgepflicht für die „Kinder“, die vielmehr mit äußerster Mißachtung behandelt werden. Korruption, im Modernen Staat ein Fremdkörper, ist dann die einzige Möglichkeit für die Untertanen, solche Apparate in Bewegung $\mathrm{zu}$ setzen ${ }^{79}$. B.-O.-Bryde's neues Buch ist das erste ${ }^{80}$, das ausschließlich diesem Thema gewidmet ist. Insgesamt bestätigt es die oben angedeutete Befürchtung: Eine gebildete Minderheit hat das Heft heute nicht anders in der Hand, als es seinerzeit die Kolonialherren gehabt haben. Bestätigt wird dieses Bild durch die Feststellung, daß es nur zwei "Idealisten" sind, die eine solche neue Schicht bekämpfen: Mao und Nyerere ${ }^{81}$.

2. Die Neuen Staaten kennen - von ganz wenigen, wenn auch nicht unwichtigen Ausnahmen abgesehen - allein die Demokratie als Staatsform ${ }^{32}$. Mag es in vorkolonialer Zeit überall eine Mitwirkung jedenfalls von Notablen an der Herrschaft

77 Vgl. z. B. Robert Laporte, Power and Privilege (sic!) - Influence and Decision-Making in Pakistan (California U.P. 1975), S. 91: „The British ,Sahib“ was replaced by the Pakistani ,Sahib'.

78 Neuerdings Joseph C. Miller, Kings and Kingsmen - Early Mbundu States in Angola - (Clarendon Press Oxford 1976).

79 Vgl. hierzu etwa J. S. Nye, Corruption and Political Development - A Cost-Benefit Analysis, in: N. T. Uphoff und W. F. Ilchmann (ed.), The Political Economy of Development (California U. P. 1973),

80 The Politics and Sociology of African Legal Development - Veröffentlichungen aus dem Institut für Internationale Angelegenheiten der Universität Hamburg, Band 2 (Frankfurt am Main 1976). Vgl. früher Internationale Angelegenheiten der Universität Hamburg, Band 2 (Frankfurt am Main 1976). Vgl. früher
schon in einzelnen Bemerkungen etwa: Patrick O'Brien, The Revolution in Egypt's Economic Svstem schon in einzelnen Bemerkungen etwa: Patrick O'Brien, The Revolution in Egypt's Economic Svstem
(Oxford U.P. 1966), S. 293: „One recent analysis of the contemporary Egyptian scene concluded that (Oxford U.P. 1966), S. 293: "One recent analysis of the contemporary Egyptian scene concluded that
a new privileged class has already emerged ... “, und: L. C. B. Gower, Independent Africa - The Challenge of the Legal Profession (Harvard U.P. 1967), S. 3, 26, 36: Die Neuen Staaten Afrikas zerfallen in zwei "distinct (oder) separated societies".

81 Vgl. Cranford Pratt, The critical phase in Tanzania 1945-1968 - Nyerere and the emergence of a socialist strategy (Cambridge U.P. 1976), S. 120.

82 Vgl. Michel Camau, La Notion de Démocratie dans la Pensée des Dirigeants Maghrébins (Paris 1971), S. $1 \mathrm{ff}$. 
von Königen oder Häuptlingen gegeben haben, so ist doch die heutige Demokratie ein übernommenes Modell. Es gibt jedoch kaum einen Staat, der diesen Typus ohne Abwandlungen eingeführt hätte - ein Umstand, der uns zu der Frage veranlaßt, ob wir es hier mit eigenständigen und eigenartigen Neubildungen zu tun haben ${ }^{83}$. Am wenigsten scheint von "Volksdemokratie“ die Rede zu sein. Hingegen haben eine gewisse Aufmerksamkeit erregt die "basic democracy" Ayubh Khans in Pakistan ${ }^{84}$ und die "guided democracy " in Indonesien ${ }^{85}$. Es sind aber weniger Abwandlungen des Typs als die Art und Weise von dessen Handhabung, die Beachtung erheischen: Ist für den Westen Demokratie gleichbedeutend mit freiheitlicher, gleichheitlicher und vor allem pluralistischer Demokratie, so sind dies Eigenschaften, die entweder den Neuen Staaten nichts sagen oder die sie bewußt durch andere ersetzen, wobei sich abermals für uns die Frage stellt, ob wir hier Ergebnisse eigener Fruchtbarkeit vor uns haben.

Der westliche Typ von Demokratie verwirklicht sich vor allem in Wahlen. Daß aber der Mensch zwischen mehreren Alternativen frei wählen kann, ist selbst in Europa eine Vorstellung, die kaum älter ist als zwei oder drei Jahrhunderte, ebenso wie ihr Zwilling, die Mobilität der Gesellschaft, insbesondere auch der Gedanke eines gesellschaftlichen Aufstieges. Man kann hiernach schwerlich erwarten, daß man in den Neuen Staaten unter "Wahl“ dasselbe vesteht wie im Westen ${ }^{85 a}$ und daß daher z. B. Wahlmanipulationen als wahlwidrig angesehen werden.

Folgerichtig muß es nicht als unerläßlich angesehen werden, daß in derWahl mehrere Parteien um die Stimmen der Wähler konkurrieren. Wäre es anders, dann hätte es nicht vorkommen können, daß nicht nur der Einparteienstaat den Vielparteienstaat in den Neuen Nationen weit überwiegt - um von Militärregimen ${ }^{86}$ gar nicht erst zu reden -, sondern daß er sich so leicht durchzusetzen und keinen geistigen Widerstand ausgesetzt zu sein pflegt ${ }^{87}$. Auf die Rechtfertigungen, die diesem Typ verliehen werden, insbesondere die verbreitete These, daß im Falle einer Mehrheit von Parteien jede sich mit einer tribalen oder lokalen Besonderheit identifizieren und daher die schwache Einheit des Staates vital gefährdet würde, braucht hier nicht eingegangen zu werden.

Erst recht aber muß es dem Denken der Neuen Staaten so gut wie unverständlich erscheinen, daß Opposition etwa Notwendiges, weil Fruchtbares ist. Erst in der zweiten Hälfte des 18. Jahrhunderts hat sich im Vaterland der freiheitlichen Demokratie, England, die Auffassung nach und nach durchgesetzt, daß Opposition nicht Hochverrat, sondern als Seiner Majestät getreue Opposition als Gegenkraft $^{88}$ notwendiger Bestandteil des politischen Prozesses einer Demokratie ist. Vor allem aber setzt die Dialektik „Regierung:Opposition“ und umgekehrt eine Gestimmtheit der Beteiligten voraus, die man durch „agonal“, fechterisch, ritterlich u. ä. m. in etwa umschreiben kann, von der selbst in

$83 \mathrm{Vgl}$. oben, S. $19 \mathrm{f}$.

84 Vgl. hierzu Mohammad Ayub Khan - Friends, not Masters - A Political Autobiography (Oxford U.P. 1967), S. $209 \mathrm{ff}$

85 Vgl. Klaus Kähler, Wirtschaftsverfassung und Kapitalinvestition in Indonesien (Beiheft 5 dieser Zeitschrift), Hamburg 1973, S. $25 \mathrm{ff}$.

85a Vgl. hierzu D. E. Apter a. a. O. (ober Anm. 50), S. 9 ff. Ferner M. Flory et R. Matran, Les Regimes Politiques des Pays Arabes (Paris 1968), S. $140 \mathrm{f}$.

86 Vgl. Herbert Krüger, Militärregime in Úbersee (1976) - Heft 8 der Beihefte zu dieser Zeitschrift.

$87 \mathrm{Vgl}$. zur Zeit gerade Indien und hierzu W. A., Indien auf dem Wege zum Einparteienstaat, in: NZZ vom 1. Januar 1977.

88 Vgl. hierzu vor allem A. S. Ford, His Majesty's Opposition (Oxford U.P. 1974). Uber das Agonale als Gestimmheit vgl. Herbert Krüger, Einheit und Freiheit - Die Strukturprobleme einer Verfassungstypologie (Hamburg 1944). 
den westlichen Ländern immer weniger zu spüren $\mathrm{zu}$ sein scheint ${ }^{89}$. Und wenn das Lebenselement dieser parlamentarischen Dialektik in der Tat die Diskussion ("government by discussion") ist, dann kann man schwerlich die Erfassung eines solchen Lebenselementes der Institution von der Dritten Welt erwarten, wenn Praxis und Verständnis im Westen selbst nicht mehr handgreiflich vorhanden sind und das Palaver ${ }^{90}$ offenbar etwas ganz anderes ist als Diskussion.

3. Ein Überblick über die Rezeption westlicher politischer Institutionen ergibt erst dann ein volles Bild, wenn man das Telos herausarbeitet, von dem sie erfüllt sind. Man ist geneigt, hierbei als erstes an "Nationalismus“ zu denken. Dieser Nationalismus ist offenbar nicht allein, wie es durchweg der Fall ist, Reaktion auf Fremdherrschaft - man hat offenbar auch die Art und Weise dieser Reaktion aus dem Westen übernommen ${ }^{91}$. Auch an dieser Stelle drängt sich wieder die Überlegung auf, ob nicht der Wegfall des Anlasses die Fortführung des Nationalismus nur auf einer neuen geistigen Grundlage möglich macht, die vielleicht eine eigenständige und eigenartige sein könnte ${ }^{92}$. Berücksichtigt man vor allem den Umstand, daß die Neuen Staaten Nationen ohne Nation sind, dann kann man den Nationalismus nicht nur als eine Vorwegnahme der Nation, sondern in ihm vor allem ein Element des „nation-building“ sehen.

Hiermit ist jedoch die Frage nach dem Telos nicht erschöpfend beantwortet. Wenn auch Kolonialherren und Kolonialvölker nicht allzu lange zusammengelebt haben, so hat dieser Zeitraum doch genügt, um die Einheimischen erkennen zu lassen, wie tief ihr Lebensstandard liegt und daß diese Tatsache offenbar nicht unabänderlich ist ${ }^{93}$. Kritik und Unzufriedenheit mit den bestehenden Zuständen und den traditionellen Herrschaftsstrukturen sind die Folge. Es mag sogar sein, daß eine solche Verstimmtheit bewußt von einheimischen revolutionären Kräften herbeigeführt ist: Folgt man nämlich Richard M. Solomon ${ }^{94}$, dann hat Mao seinen Mitbürgern die Angst vor Verwirrung (confusion) des Lebens und der Gefühle genommen, mit der der Konfuzianismus die strenge und feste Ordnung der Verhältnisse rechtfertigte, und sie demgegenüber systematisch zur Unzufriedenheit erzogen, die die Revolution letztlich hat siegen lassen.

Das Ergebnis ist in beiden Fällen der Wohlfahrtsstaat und die Genußgesellschaft das westliche Glück ${ }^{95}$. Die Übernahme dieses „Ideals“ scheint mir die entscheidende, wenn auch wohl kaum erfreuliche Wirkung des Westens auf die Neuen Staaten zu sein. Allerdings haben wir bemerken müssen, daß es bisher offenbar nur eine kleine, geschlossene Schicht ist, die sich eines solchen Glückes hat bemächtigen können, ohne es zu "demokratisieren“. Es ist früher erwähnt worden, daß es lediglich zwei Staatsmänner sind, die sich gegen ein solches Ergebnis wenden: Mao

89 So geht z. B. Hans-Peter Schneider, Die parlamentarische Opposition im Verfassungsrecht der Bundesrepublik Deutschland I (Frankfurt am Main 1974), auf diese Voraussetzung, das "Temperament ${ }^{\star}$ des Instituts nicht ein.

$90 \mathrm{Vgl}$. Joseph Kasule, Palaver and its influence in current constitutional and international law - Kölner Juristische Dissertation 1972.

$91 \mathrm{Vgl}$. Leonard Binder, Ideological Foundations of Egyptian - Arab Nationalism, in dem oben, Anm. 68, bezeichneten Sammelwerk, S. 128 ff., S. 133.

92 Vgl. Georges Burdeau, Traité de Science Politique III (2. Aufl., Paris 1969), S. 159: „Cessant d'être la réaction à l'humiliation de l'opprimé, elle est devenue forme de l'orgueil des pauvres“.

93 Hierzu Karl von Vorys, Political Development in Pakistan (Princeton U.P. 1965), S. $83 \mathrm{ff}$

94 Mao's Revolution and the Chinese Political Culture (Califormia U.P. 1971). Es bedarf kaum der Erwähnung, daß die Kritik diese These als grundfalsch bezeichnet hat; vgl. F. W. Mote, China's Past in the Study of China Today - Some Comments on the Recent Work of Richard Solomon, in: (Ich verdanke die Kenntnis dieser Kritik der Freundlichkeit Wolfgang Frankes).

95 Vgl. hierzu aber A. M. Kamarck, The Economics of African Development (New York usw. 1967), S. 50: "This does not mean that the traditional Africa has the same almost unlimited desire for material goods that the modern America has". 
und Nyerere. Darüber hinaus mag die Möglichkeit bestehen, daß das Bekenntnis zu "Sozialismus“ nicht nur den westlichen Kapitalismus - Imperialismus verneinen soll - daß es sich vielmehr auch und vor allem gegen die Ideale wendet, die mit „Wohlfahrtsstaat" und "Genußgesellschaft" bejaht sind.

Damit ist die Aufgabe für eine gelegentliche Fortsetzung dieser Untersuchung gestellt: Sie wird vor allem zu ergründen haben, was diejenigen Neuen Nationen, die sich irgendeinem "Sozialismus" verschrieben haben, hierunter verstehen, insbesondere ob es sich lediglich um eine Rezeption aus dem Osten handelt; und ob sie solchen ihren Sozialismus dem Wohlfahrtsstaat und der Genußgesellschaft entgegensetzen: Ließe sich das Letzte bestätigen, dann wäre damit zugleich die Möglichkeit einer Rezeption aus dem Osten verneint, da dieser gerade auf solche Ideale hinstrebt ${ }^{97}$. 


\title{
The Ideological Orientation of the New States
}

\author{
BY Herbert Krüger
}

The ideological orientation of the new states can derive from their own values and traditions or from an intellectual exchange with "East" and "West". After the end of colonialism, the former attitude could be expected, but the new states' desire to "modernize" turns their view to the examples of East and West. It is, however, doubtful whether these old continents have much to offer, as they are themselves in need of renewal and their rigid, quasireligeous ideologies are not too inviting for countries looking for new ways. Despite these shortcomings of East and West, there are only few countries attempting a complete return to their own roots. Most of the time, authenticity is restricted to superficialities like names. More important are attempts at a "Third Way". The various organizations establishing co-operation among the developing countries which have made their impact in International Law and ideologies like "Arab", "African" etc. . . "Socialism" are important in this respect. With regard to intellectual communication between the West and the Third World, there are not many Western theoreticians with major impetus (Marx being introduced as an representative of "Eastern" thought). Comte was influential in Latin America, Bentham in India, but on the whole, the West has been more successful in exporting institutions rather than ideologies. The most important such institution is the "state", though in many countries of the Third World it has become the instrument of a small elite instead of an institution of general welfare.

The same is true for "democracy", to which almost all countries pay lip-service without adopting such essential elements as free elections between alternatives, freedom of opposition (elements which also in the West needed centuries to develop). A western heritage of doubtful value, finally, is the pursuit of welfare and affluence, monopolized by a small elite but aspired to by all. It remains to be studied, how systems subscribing to socialism and austerity handle these problems, and how much they are influenced by the "East" or going their own way.

\section{The State Class/Administrative Bourgeoisie in Underdeveloped}

\section{By Hartmut Elsenhans}

The developing countries will be able to appropriate the differantial rents and the consumer rents from their raw material exports (and later on from their exports of simple processed articles). Overcoming underdevelopment will no longer be hampered by lack of foreign currency or investible funds, but nevertheless will not be possible automatically by the disposal of funds. Under this assumption the internal societal dynamics of underdeveloped countries become a problem of primordial concern.

The preceding article tries to give new hints for dealing with the tendencies of increasing state participation in economic development of the LDC's. Considering that the sharpening social crisis in the LDC's forces both governments which 